Design: Determinants of Adolescent Social well-being and Health (DASH) study: A cohort observational study.

Setting: Schools in ten London boroughs with high proportions of the main ethnic minority groups, UK.

Participants: White British (692), Black Caribbeans (670), Black Africans (772), Indians (384), and Pakistanis and Bangladeshis (402) children were surveyed in 2003/2004 (11-13 years) and followed up in 2005/2006 (14-16 years).

Main outcome measures: Longitudinal measures of systolic (sBP) and diastolic BP (dBP). Predicted age, gender and ethnic specific mean BP, adjusted for anthropometry (Body mass index (BMI), height, weight, leg length (LL)), smoking, socio-economic circumstances (SEC) and psychological well-being were derived using mixed models.

Results: Among boys, sBP did not differ by ethnicity at $12 \mathrm{y}$ but the greater increase among Black Africans than Whites led to higher $\mathrm{sBP}$ at $16 \mathrm{y}(+2.9 \mathrm{~mm} \mathrm{Hg})$. The age trends for dBP suggest earlier divergences and increasing disparities with significantly higher $\mathrm{dBP}$ than Whites from 12 y for Indians, 14 y for Pakistanis-Bangladeshis and from $15 \mathrm{y}$ for Black Africans. Among girls, ethnic differences in mean sBP were not significant at any age but larger increases were observed for Black Caribbeans and Black Africans than for Whites. At $12 \mathrm{y}, \mathrm{dBP}$ was lower among Black Caribbean and African girls than White girls but the faster rise led to similar levels by $14 \mathrm{y}$. Indians had significantly higher $\mathrm{dBP}$ from $13 \mathrm{y}$ and Pakistanis/ Bangladeshis from $15 \mathrm{y}$. Body mass index, height and leg length were independent predictors of BP, with few ethnic specific effects. Socio-economic disadvantage had a disproportionate effect on BP for girls in minority groups.

Conclusions: The findings suggest that ethnic divergences in $\mathrm{BP}$ begin in adolescence, particularly striking for boys. They signal the need for early prevention of adverse CVD risks in later life.

\section{NUTRITIONAL COMPOSITION OF THE DIETS OF SOUTH ASIAN, BLACK AFRICAN CARIBBEAN AND WHITE EUROPEAN CHILDREN IN THE UK: THE CHILD HEART AND HEALTH STUDY IN ENGLAND}

${ }^{1}$ AS Brock, ' $\mathrm{CM}$ Nightingale, ' $\mathrm{CG}$ Owen, ${ }^{1} \mathrm{AR}$ Rudnicka, 'MC McNamara, ${ }^{2} \mathrm{CJ}$ Prynne, ${ }^{2}$ AM Stephen, 'DG Cook, 'PH Whincup. 'Division of Community Health Sciences, St George's, University of London, London, UK; ${ }^{2}$ Medical Research Council Human Nutrition Research, Cambridge, UK

\section{doi:10.1136/jech.2009.096727g}

Background: Compared with UK white Europeans, South Asian adults have increased risks of obesity, type 2 diabetes, stroke and coronary heart disease (CHD); black African-Caribbean adults have increased risks of obesity, type 2 diabetes and stroke with lower $\mathrm{CHD}$ risk. Dietary differences could be important in explaining these ethnic differences, which appear to emerge in early life. However, few systematic attempts have been made to define the extent of ethnic differences in diet, particularly in children.

Objective: To examine ethnic variations in nutritional composition of the diets of children of South Asian, black African-Caribbean and white European origin in the UK.

Design: 24 hour recalls of dietary intake were carried out during a cross-sectional survey of children attending 85 Primary Schools in London, Birmingham and Leicester.

Participants: 2210 children aged 9-10 years, including 567 of South Asian, 595 of black African-Caribbean and 601 of white European origins.

Results: Compared to white Europeans, South Asian children reported higher mean total energy intake (mean difference $96 \mathrm{kcal}$, 95\% CI $35157 \mathrm{kcal}$ ), fat \% energy (mean difference 1.3\%, 95\% 0.5 to $2.1 \%$ ) and protein \% energy (mean difference $0.9 \%, 95 \%$ CI 0.5 to $1.3 \%)$. Their intakes of carbohydrate as a proportion of energy (particularly sugars), vitamin $\mathrm{C}$ and $\mathrm{D}$, calcium and haem iron intakes were lower. These differences were especially marked for Bangladeshi children. Black African Caribbean children had lower intakes of total fat \% energy (mean difference $-1.3 \%, 95 \%$ CI -2.0 to -0.5 ) and saturated fat $\%$ energy (mean difference $-1.2 \%, 95 \%$ CI -1.6 to -0.8$)$. They also consumed less non-starch polysaccharide, vitamin $\mathrm{D}$ and calcium; whilst intakes of haem iron were higher. The lower intakes of fat (including saturated fat) were particularly marked among black African children.

Conclusions: Appreciable ethnic differences exist in the nutritional composition of children's diets. These patterns could influence early emerging differences in disease risk and, if maintained, could contribute to continuing ethnic differences in disease risk in the next generation.

\section{PATTERNS OF ADIPOSITY AND OBESITY AMONG SOUTH ASIAN AND WHITE EUROPEAN CHILDREN: CHILD HEART AND HEALTH STUDY IN ENGLAND}

CM Nightingale, AR Rudnicka, CG Owen, DG Cook, PH Whincup. Division of Community Health Sciences, St George's, University of London, London, UK

\section{doi:10.1136/jech.2009.096727h}

Background: The prevalence of obesity is increasing markedly both in UK adults and children. Risks of obesity and its consequences (particularly type 2 diabetes) are particularly marked among South Asians. Earlier studies have suggested that adiposity patterns in Asian populations differ from those in Europeans, making standard weight-for-height measures misleading. However, few studies have made detailed assessments of adiposity and obesity among UK South Asian and white European children.

Objective: To examine ethnic differences in adiposity/obesity patterns and their inter-relations in UK children of South Asian and white European origin.

Design and Methods: Cross-sectional survey of children (Child Heart and Health Study in England (CHASE)) recruited from a sample of 200 Primary schools with high prevalences of children of South Asian origin in London, Birmingham and Leicester. Standardised measurements of anthropometry (weight, height, waist circumference, multiple skinfolds) and of body fat $\%$ by bioelectrical impedance were made. Ethnic origin was defined primarily by parental self-report. Statistical analyses were adjusted for age and gender and included a random effect to allow for clustering within schools. All body size measurements were log transformed with the exception of body fat \%. Obesity cut-offs were defined using International Obesity Task Force guidelines.

Participants: 5759 children aged 9-10 years, of whom 1490 were of white European and 1543 were of South Asian origin (overall response rate $68 \%$ ).

Results: South Asian children were lighter (\% difference - 2.7, 95\% CI -4.3 to -1.0 ), had a lower body mass index (\% difference -2.2 , $95 \%$ CI -3.5 to -0.9 ) and a lower waist circumference (\% difference $-1.5,95 \%$ CI -2.5 to -0.5$)$ than white Europeans; there was no appreciable difference in height. However, South Asian children had a higher body fat $\%$ (mean difference $1.7,95 \%$ CI 1.0 to 2.4 ) and a higher combined skinfold thickness (\% difference 5.1, $95 \%$ CI 1.4 to 9.0). For a given BMI, mean body fat \% among South Asians was $2.3 \%$ (95\% CI $2.0 \%$ to $2.7 \%$ ) higher; this difference varied only slightly by gender and ethnic subcategory. The unadjusted prevalence of obesity based on body mass index was $0.1 \%$ lower among South Asians than white Europeans, but when based on body fat \% was $9 \%$ higher.

Conclusions: Patterns of adiposity/obesity differ between UK children of South Asian and white European origin and may have adverse long-term health consequences. However, the differences are not well represented by simple measures based on weight and height alone. 